\title{
DEVALUACIONES REALES Y ACELERACIONES INFLACIONARIAS ${ }^{\circ}$
}

\author{
REAL DEVALUATIONS AND INFLATION ACCELERATIONS \\ Pablo Carreras Mayer*
}

enviado: 25 marzo 2017 - aceptado: 24 abril 2017

\begin{abstract}
Resumen
Las economías abiertas pueden enfrentar un trade-off entre aumentos en el tipo de cambio real (TCR) y cambios en la inflación para horizontes relevantes. Se aplica una metodología a un panel mensual de 98 países para el período 1970-2004 identificando episodios de incrementos "significativos y duraderos" del TCR, clasificándolos en "aceleradores" o "no aceleradores" según conlleven cambios apreciables en la tasa de inflación. Se aprecia elevada heterogeneidad en la respuesta inflacionaria entre regiones y entre países emergentes y avanzados. La probabilidad de episodios "aceleradores" (i) se incrementa a mayor inflación inicial, (ii) no resulta influida por la brecha de producto, (iii) disminuye con la presencia de regímenes de Inflation Targeting, y (iv) disminuye a mayor grado de "anclaje cambiario" posterior al episodio.
\end{abstract}

Código JEL: F41, E31, F31.

Palabras clave: macroeconomía abierta, inflación, tipo de cambio.

* Universidad de Buenos Aires (UBA) y Centro de Estudios de Estado y Sociedad (CEDES), Buenos Aires, Argentina. Correo electrónico: pcarrerasmayer@gmail.com.

Este artículo resultó ganador del premio Estudios Económicos en la octava edición del Congreso Nacional de Estudiantes de Postgrado en Economía (CNEPE) 2017. La versión en línea de este artículo cuenta con material adicional.

- Carreras Mayer, P. (2017). Devaluaciones reales y aceleraciones inflacionarias. Estudios económi$\cos , 34(68), 39-73$. 


\begin{abstract}
Open economies may experience trade-offs between increments in the real exchange rate (RER) and inflation accelerations lasting throughout relevant time horizons. A methodology is applied on a monthly panel comprising 98 countries during 19702014. Episodes of "significant and extended" increases of the RER are identified, classifying them as "accelerating" or "non-accelerating" according to inflation dynamics. The inflationary response of the regions under study is highly heterogenous, as well as between emerging and advanced economies. The probability of an "accelerating" episode (i) increases the higher the initial inflation rate, (ii) is not affected by the output gap, (iii) diminishes under Inflation Targeting regimes, and (iv) diminishes the stronger is the nominal exchange rate anchor after the devaluation.
\end{abstract}

JEL Code: F41, E31, F31.

Keywords: Open Economy Macroeconomics, Inflation, Foreign Exchange.

\title{
INTRODUCCIÓN
}

La historia económica argentina muestra repetidos episodios de subas significativas y "duraderas" del tipo de cambio real (TCR) que dieron lugar a aceleraciones en la inflación. Eventos de este tipo se han observado también en otros países de la región y el mundo, aunque con menor frecuencia (Frenkel y Rapetti, 2010; Ito y Sato, 2008). La ocurrencia de estos episodios sugiere la existencia de un trade-off entre el incremento del TCR y la "estabilidad" (en el sentido de no-aceleración) de la tasa de inflación.

La literatura reciente señala dos fenómenos que parecieran indicar que esta relación no ha sido estable a lo largo del tiempo. Por un lado, diversos estudios sobre el traspaso de variaciones en el tipo de cambio nominal (TCN) a la tasa de inflación indican que este ha disminuido a lo largo del tiempo (Frankel, Parsley y Wei, 2012). Otros trabajos se han focalizado en el análisis de episodios recientes de grandes devaluaciones destacando que estas no han involucrado alteraciones significativas en las tasas de inflación (Burstein, Eichenbaum y Rebelo, 2005).

Este trabajo se propone aportar en esta línea de investigación. Analizando empíricamente la frecuencia de ocurrencia de este trade-off, se procura indagar sobre las condiciones comunes a los episodios que generan que las subas "duraderas" del TCR conduzcan a períodos de aceleración inflacionaria. 
Se analiza un panel para 98 economías en el período 1970-2014, identificando episodios de devaluación real "significativos y duraderos". Luego se distingue entre aquellos que presentan una aceleración de la tasa de inflación (episodios "aceleradores") y aquellos que no presentan aceleración (episodios "no-aceleradores"). La tasa de inflación de interés para este trabajo es la variación del IPC acumulada a 12 meses.

Se encuentra que existe una gran heterogeneidad en la respuesta inflacionaria para las diversas regiones bajo estudio y entre los países "emergentes" y "avanzados". Asimismo, la respuesta difiere marcadamente entre países con una historia cambiaria referenciada al dólar (USD) y aquellos que no².

Los resultados obtenidos sugieren que la ocurrencia de episodios "aceleradores" está asociada: (i) positivamente a la tasa de inflación inicial, (ii) negativamente al grado de "anclaje cambiario" posterior al episodio devaluatorio (i.e. aquellos casos que fijan el TCN presentan menor frecuencia de casos "aceleradores"), y (iii) negativamente a la existencia previa de un régimen de política monetaria de Inflation Targeting (IT). Adicionalmente, no se encuentra una relación robusta entre la brecha de producto y la ocurrencia de estos episodios.

Siguiendo esta introducción, el trabajo se estructura como sigue. En la sección I se discuten las condiciones bajo las cuales un cambio duradero en el TCR puede desencadenar un episodio acelerador. En la sección II se presenta la metodología de identificación de los episodios de devaluación real, y el método de clasificación entre episodios "aceleradores" y "no aceleradores". Luego de revisar los hechos estilizados, en la sección III se discute la estrategia econométrica empleada para estudiar la frecuencia de los episodios "aceleradores" y se presentan los resultados obtenidos. Finalmente, el trabajo concluye con unas breves reflexiones.

\section{INFLACIÓN EN ECONOMÍA ABIERTA Y PEQUEÑA}

En una economía abierta, un índice general de precios domésticos (por caso el IPC) puede descomponerse en precios transables y no transables. Los bienes no

1 De acuerdo con la clasificación por nivel de ingreso del Banco Mundial, se denomina avanzados a aquellos que pertenecen a la categoría de altos ingresos (high-income), mientras que los categorizados como emergentes son todos los demás.

2 Los países que utilizan el USD como referencia surgen del trabajo de Shambaugh (2004). 
transables son del tipo fix (Hicks, 1989), mientras que entre los bienes transables podemos distinguir entre exportables e importables.

Bajo la tradición "neo-estructuralista", la dinámica de la inflación se estudia a partir del comportamiento de algunos precios clave que componen el índice general de precios. La tasa de inflación, en el corto plazo, resulta del comportamiento de este conjunto de precios. En este marco, se asume que los precios, y en particular los salarios, son inflexibles a la baja (Olivera, 1967).

En el caso de los bienes fix, sus precios son fijados y mantenidos por un período relevante aplicando un mark-up sobre los costos medios "normales". Estos costos incluyen el pago de salarios, y otros servicios contratados como alquileres, así como la utilización de insumos importados en el proceso productivo.

Por su parte, tradicionalmente se asume que los precios de los bienes transables se fijan siguiendo la ley de un solo precio (Froot y Rogoff, 1995), con lo cual resultan idénticos a los precios internacionales expresados en moneda doméstica. No obstante, el cumplimiento de la ley requiere (i) ausencia de aranceles u otros impuestos ad-valorem, y (ii) competencia perfecta.

Si se asume que los precios internacionales son constantes, que la tecnología se modifica lentamente en el tiempo y que el mark-up es un factor estructural determinado por las condiciones de mercado, puede mostrarse que la tasa de inflación depende principalmente de la variación del TCN y de los salarios.

En este marco, la ocurrencia de "episodios aceleradores" ante cambios en el TCR está sujeta a dos condiciones. Primero, las devaluaciones deben generar una suba del nivel general de precios a partir de su impacto directo sobre los bienes transables que componen el índice o que forman parte de la estructura de costos de los bienes fix. Llamamos a esto efecto impacto, o de primera ronda.

Segundo, la suba inicial en el nivel de precios debe desatar subas de otros precios no afectados en forma directa por la suba del TCN. Podemos llamar a estas subas efectos de segunda ronda. Esto está íntimamente ligado al funcionamiento del mercado de trabajo y el comportamiento que presenten los salarios nominales. 


\section{I.1. Efectos de primera ronda}

El impacto directo de una devaluación debería manifestarse (i) a través de los precios transables que componen el índice, y (ii) por la incidencia de los costos transables en la producción de los bienes y servicios no transables.

Si los precios de los bienes transables se fijan de acuerdo con la ley de un solo precio el pass-through a los precios transables es completo (Feenstra y Kendall, 1997). No obstante, la evidencia a favor del cumplimiento de la ley es débil. Resulta frecuente que el pass-through sea incompleto (Aron, Macdonald, y Muellbauer, 2014). La explicación de este fenómeno suele asociarse con la presencia de márgenes variables que absorben las variaciones cambiarias, producto de diversas estrategias de las firmas al estilo de pricing-to-market devenidas de entornos de competencia imperfecta (Goldberg y Hellerstein, 2008). Adicionalmente, podría resultar factible que los bienes fix ajusten su margen, absorbiendo los incrementos en el costo de los insumos.

La evidencia parece indicar que en los últimos 20 años, el pass-through se ha moderado, tanto en los países desarrollados como en los países emergentes (Frankel et al., 2012). Entre las principales hipótesis que dan cuenta de este cambio estructural se señala (i) el rol de la política monetaria, que ha tendido a consolidar un entorno de inflación baja y estable (Taylor, 2000) y (ii) un incremento de la competencia en las cadenas de comercialización, haciendo que los productores e intermediarios tiendan a absorber las fluctuaciones cambiarias (Bacchetta y Wincoop, 2003) $)^{3}$.

\section{I.2. Efectos de segunda ronda}

La aceleración inflacionaria que provocan los efectos de primera ronda puede resultar un fenómeno transitorio. Una vez absorbidos sus efectos, el ritmo inflacionario podría retornar al valor anterior al episodio. No obstante, una dinámica de este estilo requiere de una caída del salario real.

3 Se puede conjeturar que el peso de los componentes no transables, al interior del índice o como parte del precio de los transables (servicios de logística y comercialización, alquileres, etc.) podría haberse incrementado en el tiempo, lo que genera que decrezca la importancia de los efectos de primera ronda. Como se discute en Burstein et al. (2005), en el marco de grandes devaluaciones, la dinámica inflacionaria subsecuente se relaciona principalmente con el comportamiento de estos componentes no transables. 
La clave de los efectos de segunda ronda está en el funcionamiento del mercado de trabajo y el proceso de fijación de los salarios. Si los asalariados demandan una recomposición de sus ingresos, el salto de precios asociado a los efectos de primera ronda podría generar una tasa de inflación "permanentemente" más elevada. Se denomina a este fenómeno "real wage resistance" (Dornbusch, 1980).

Bajo una formulación bastante general, el comportamiento en el tiempo de los salarios responderá a (i) las expectativas de inflación, (ii) las condiciones del mercado de trabajo reflejadas en los niveles de desempleo y (iii) cambios regulatorios e institucionales que afecten la capacidad de negociación sindical (e. g. salario mínimo).

Que los salarios se fijan en función de la inflación refleja que los trabajadores en última instancia negocian bajo una meta de salario real (Olivera, 1991; Vera, 2014). Los efectos de los factores institucionales y el nivel de desempleo en el proceso de variación de los salarios pueden fundamentarse desde los modelos de "negociación" (McDonald y Solow, 1981) o los modelos de salarios de eficiencia (Akerlof y Yellen, 1986).

El impacto inflacionario asociadas a aumentos en el TCR no genera una aceleración de la tasa de inflación solo en los casos en que disminuyen las expectativas de inflación de los asalariados, o se deteriora la capacidad de negociación por aumentos en el desempleo y cambios institucionales, disminuyendo el salario real de equilibrio.

Si estas condiciones no se cumplen, el incremento en el TCR genera una aceleración en la tasa de inflación. La misma implicará un cambio transitorio (o no) del proceso inflacionario dependiendo el mecanismo de formación de expectativas o, de forma más general, el mecanismo de actualización de los salarios reales. A los efectos de este trabajo, interesa discutir al menos tres posibilidades: (i) expectativas adaptativas (Phelps, 1969), (ii) indexación a la inflación pasada (Frenkel, 1986) y (iii) expectativas "ancladas" por reglas de política monetaria (Barro y Gordon, 1983).

En el primer caso, las expectativas de inflación siguen un proceso del tipo $\pi_{t}^{e}=\theta \pi_{t-1}+(1-\theta)\left(\pi_{t-1}^{e}-\pi_{t-1}\right)$, siendo , por el cual los agentes revisan período a período su estimación de inflación en función de los errores de pronóstico. Si el coeficiente de actualización o aprendizaje $(\theta)$ es relativamente elevado (tendiendo a la unidad), un impulso transitorio por efecto de la devaluación im- 
plicará un cambio sostenido en la tasa de inflación, ya que el shock se incorporará rápidamente en las expectativas.

Bajo esta especificación el proceso inflacionario exhibe una fuerte inercia, y los cambios al alza en precios relativos inducen aceleraciones en la tasa de inflación. El impulso inflacionario asociado al shock cambiario, y su propagación en el tiempo, solo podría ser contrarrestado por un cambio en la dinámica de los salarios asociada a (i) aumentos en el desempleo o (ii) cambios regulatorios e institucionales que disminuyan el poder de negociación de los trabajadores.

La presencia de indexación (formal o informal) a la inflación pasada puede constituir también una fuente de inercia para el proceso inflacionario, aunque su base teórica es diferente al mecanismo de expectativas adaptativas.

La indexación responde al entorno inflacionario. En regímenes de inflación elevada, el mecanismo indexatorio constituye una respuesta óptima de los agentes para reducir costos de renegociación de contratos y adaptarse al entorno de elevada volatilidad nominal (Frenkel, 1989; Heymann y Leijonhufvud, 1995). Estos mecanismos de indexación presentan períodos de reajuste endógenos que responden a, entre otras variables, el propio nivel de inflación. Por esta razón, esperaríamos que la posibilidad de un cambio permanente en la tasa de inflación luego de un shock al TCR dependa de los niveles de inflación antes de la devaluación (Frenkel, 1989).

Muchos modelos señalan que los trabajadores fijan el salario nominal en función de un salario real "aspirado" o meta, y desarrollan mecanismos de renegociación que buscan recuperar el pico de salario real asociado a dicha meta (Olivera, 1991; Vera, 2014). Ante la presencia de indexación en el mercado de trabajo se obtiene un resultado típico de los modelos estructuralistas: todo ajuste de precios relativos (de transables flex con respecto a no transables fix) acelera la tasa de inflación (Heymann, 1986).

Pasamos a considerar el último caso de interés: expectativas inflacionarias ancladas. Asumiendo que el gobierno o el Banco Central tiene control sobre la tasa de inflación por medio de instrumentos de política, y el público tiene pleno conocimiento de esto, las expectativas de inflación se basan en el comportamiento esperado de la autoridad monetaria (Barro y Gordon, 1983). Las autoridades pueden comprometerse a una regla de política que vincule instrumentos con objetivos. Si la regla resulta "creíble", i. e. consistente con los incentivos reputacionales o de costos, las expectativas de inflación del público deberían igualarse al objetivo de 
la autoridad monetaria. En este caso, esperaríamos que los asalariados negociaran salarios bajo una expectativa, donde representa el objetivo fijado por el Banco Central.

En este caso, los efectos de aceleración inflacionaria de la devaluación serían de corta duración y explicados únicamente por los efectos de primera ronda.

\section{I.3. Las respuestas de política económica y la influencia del régimen}

Ocurrida la devaluación real, es factible que el gobierno aplique una política monetaria contractiva con el objetivo de limitar el impacto inflacionario. En una economía relativamente abierta y financieramente integrada, la política monetaria afecta a la inflación a través de tres canales: expectativas, demanda agregada, y por su impacto sobre el TCN ${ }^{4}$. Los primeros dos canales ejercerán su influencia principalmente a través de la dinámica de los salarios, mientras que el tercero afectará directamente a los bienes transables e indirectamente a los no transables, a través de la estructura de costos.

El canal de expectativas, discutido implícitamente antes, hace referencia a la capacidad que tiene el Banco Central de orientar las expectativas de inflación de los formadores de precios, tanto por medio de anuncios como de forma implícita a través de sus acciones. Si los agentes confían en la capacidad del mismo de cumplir con la meta anunciada (i.e. si goza de credibilidad y reputación), fijarán precios bajo esta pauta y la inflación convergerá a la meta.

El canal de demanda agregada se asocia a la influencia que los cambios en la tasa de interés y el volumen de crédito generan sobre las decisiones de gasto del sector privado. El impacto sobre los precios es indirecto: un aumento en las tasas de interés genera una caída en el gasto agregado, y esto tiende a moderar las subas de precios y salarios por efecto de las menores ventas y los aumentos en el desempleo.

4 Esta clasificación de los canales constituye una simplificación a meros efectos expositivos. Por ejemplo, dentro del canal de "demanda agregada" estamos incluyendo tanto al canal de tasas de interés, como a los canales de crédito, y los efectos riqueza que generan cambios en la demanda agregada a partir de la modificación de las tasas de corto plazo. Una discusión más detallada de los mecanismos de transmisión de la política monetaria puede consultarse en Agénor y Montiel (2008). 
Al modificar los rendimientos de los activos domésticos, la política monetaria induce a los agentes a cambiar la composición de su portafolio de activos. En consecuencia, un incremento (disminución) de la tasa de interés doméstica tiende a estabilizar o apreciar (depreciar) el TCN. Esto opera en el proceso inflacionario ejerciendo influencia en los precios de los bienes finales - exportables o importables- y sobre el costo de los insumos importados.

El propio régimen de política monetaria puede influir en las expectativas de inflación de largo plazo. Esta es la idea detrás de los regímenes de "Inflation Targeting" (IT). La adopción de este esquema podría generar (i) que los impulsos transitorios producto de la devaluación no se perpetúen en el tiempo (Reyes, 2007), y (ii) que el impacto inmediato del TCN tienda a disminuir (Taylor, 2000).

Finalmente, la continuación del impulso inflacionario podría verse influido por el régimen cambiario. La introducción de un régimen cambiario como instrumento para controlar la tasa de inflación ha sido una práctica usual en la historia latinoamericana. Existe gran cantidad de experiencias donde se ha utilizado al TCN como ancla nominal para detener el proceso inflacionario, ya sea fijando el mismo o bajo un esquema de "crawling peg activo" (Frenkel y Rapetti, 2010).

\section{EPISODIOS DE DEVALUACIÓN REAL Y ACELERACIÓN INFLACIONARIA}

\section{II.1. Datos utilizados}

El panel utilizado para detectar los episodios de devaluación real abarca 98 economías, emergentes y desarrolladas, durante el período 1970-2014. La fuente principal es la base International Financial Statistics (IFS) compilada por el FMI 5 .

Para analizar la probabilidad de ocurrencia de episodios aceleradores se construye una base de tipo "corte transversal" incorporando variables adicionales a inflación y TCR. Fuentes y metodología de construcción disponibles en el apéndice (tabla A1).

5 En algunos casos, para ganar una mayor cobertura temporal, se decidió reemplazar los IPC de carácter nacional reportados en el IFS por IPC de áreas urbanas, publicados por los institutos nacionales de estadística de cada país. Este es, por ejemplo, el caso de Venezuela, donde se utiliza el IPC metropolitano de Caracas en lugar del IPC de cobertura nacional. 
II.2. Criterio de identificación de episodios devaluatorios

La metodología aplicada extiende la estrategia de identificación utilizada por Bussière, Saxena y Tovar (2012) para identificar crisis cambiarias. A diferencia de esta metodología, se opta por parametrizar los umbrales de variación, eliminando la necesidad de adoptar múltiples "definiciones" para los episodios.

El TCR bilateral del país j para el mes t se define como:

$$
q_{j, t}=\frac{E_{j, t}^{U S D} P_{t}^{E E U U}}{P_{j, t}}
$$

Donde $E_{j, t}^{U S D}$ es el TCN bilateral de j contra el USD, $P_{t}^{E E U U}$ es el IPC de Estados Unidos, y $P_{j, t}$ es el IPC del país j.

Se considera que se produce un episodio de depreciación real "significativo y duradero" cuando se cumplen, simultáneamente, tres condiciones:

1. El TCR $\left(q_{t}\right)$ en un determinado mes $(\mathrm{t})$ exhibe una variación trimestral acumulada superior a un umbral mínimo $\psi>0$. En términos formales:

$$
\frac{q_{t}-q_{t-3}}{q_{t-3}} \geq \psi
$$

2. La variación en el TCR debe estar asociada a un cambio significativo en el TCN (al menos 2/3 de $\psi$ ), descartando casos donde la depreciación es un resultado de la dinámica de precios domésticos vis a vis los precios internacionales.

3. El TCR, luego de transcurrido un lapso temporal de longitud $H_{q}$ (i.e. 12 , 24, o 36 meses luego del episodio), debe mantenerse en niveles "por encima" de los que exhibía antes de ocurrido el episodio:

$$
\frac{q_{t+H q}}{q_{t-3}} \geq \lambda \quad \text { con } \lambda \geq 1
$$

En resumen, este algoritmo permite detectar episodios de aumentos en el TCN que implican cambios en el TCR en, al menos, una magnitud $\psi$ y que al cabo de $H_{q}$ meses de ocurrido todavía presentan un nivel al menos igual al que exhibían antes de la devaluación. Se analiza la variación en el TCR a lo largo de un trimestre para contemplar casos donde la devaluación presenta una dinámica más suave que la de un salto discreto en un mes. No obstante, bajo el criterio propuesto hay 
casos en los cuales varios meses seguidos cumplen con las condiciones descritas. Para asociar el shock cambiario a un único mes se fija la ocurrencia del episodio en el mes donde la tasa de devaluación nominal trimestral acumulada es máxima.

\section{II.3. Episodios de devaluación real identificados}

La tabla 1 reporta la cantidad de episodios de devaluaciones reales persistentes para un horizonte de evaluación de doce meses $(H q=12)$. Se presenta la sensibilidad en la identificación de episodios para distintos valores de los dos parámetros principales del algoritmo. Se aprecia que la condición ligada al umbral mínimo de variación trimestral de $\mathrm{q}(\psi)$ resulta el parámetro más restrictivo para identificar episodios. Para lo que resta del trabajo optamos por fijar $\lambda=1$. Asimismo, en aras de simplificar la exposición de los resultados se fija el valor del horizonte $H q$ en 12 meses.

Tabla 1. Cantidad de episodios de depreciación identificados bajo distintas combinaciones de los parámetros

\begin{tabular}{ccccccc}
\hline & & \multicolumn{5}{c}{ Variación trimestral acumulada en . } \\
\cline { 2 - 7 } & & 0.05 & 0.10 & 0.15 & 0.20 & 0.25 \\
\cline { 2 - 7 } Persistencia & 1.000 & 1454 & 571 & 266 & 138 & 105 \\
del cambio de $q$ & 1.025 & 1332 & 546 & 258 & 134 & 100 \\
& 1.050 & 1193 & 513 & 245 & 132 & 97 \\
& 1.075 & 1050 & 478 & 233 & 129 & 95 \\
\cline { 2 - 7 } & 1.100 & 933 & 445 & 208 & 125 & 94 \\
\hline
\end{tabular}

Fuente: elaboración propia sobre la base del IFS (FMI).

Se observa una cantidad aproximadamente equivalente de eventos por década $^{6}$. No obstante, se aprecian grandes concentraciones de eventos en torno a años particulares (figura 1). En general, estos años coinciden con eventos de crisis internacionales y recesiones en las economías desarrollada. Por ejemplo, la crisis del 2008-09, la crisis de deuda de los 80 de Latinoamérica, y las crisis de fines de los 90 en los emergentes.

6 Con excepción de la década de 1970, donde, por cuestiones de disponibilidad de información, el panel presenta una dimensión más reducida. 
Figura 1. Episodios de devaluación real

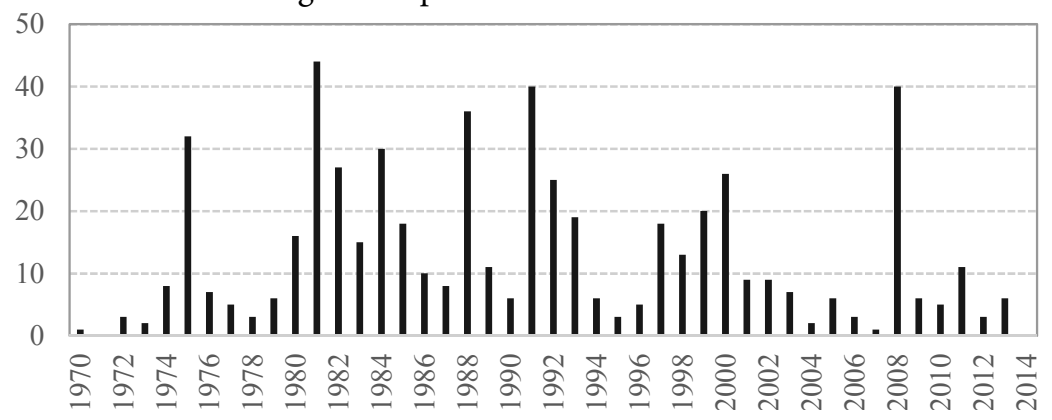

Nota: parámetros de identificación $\psi, \lambda$, y Hq.

Fuente: elaboración propia sobre la base del IFS (FMI).

La mayoría de los episodios se concentran en entornos inflacionarios con tasas de hasta un $30 \%$ anual (promedio de los 6 meses previos), rasgo que se mantiene aun cuando consideramos solo devaluaciones de mayor tenor.

La figura 2 muestra la distribución de la variación acumulada de la tasa de inflación en torno a los episodios de devaluación detectados. En todos los casos, se toma la variación porcentual respecto de la tasa de inflación que presentaba la economía en t-3, donde t se refiere al mes en el cual se identifica la ocurrencia del episodio de devaluación. De esta manera, podemos analizar la dinámica normalizando en función a la inflación previa a la ocurrencia del shock.

Cuando consideramos el total de episodios, se destaca que más del $50 \%$ de los casos evidencia una aceleración en la tasa de inflación, si bien de magnitudes variables. Esto muestra que, en buena medida, un shock de precios relativos tiende a afectar el nivel general de precios; lo que en versión "dinámica" resulta equivalente a un incremento en la tasa de inflación. Esta conclusión se traslada también cuando consideramos el caso de los avanzados y los emergentes (paneles b y c). No obstante, surgen nítidamente algunas diferencias. En el caso de los emergentes, la distribución es más asimétrica y las magnitudes de aceleración son mayores.

Asimismo, se destaca que, en una proporción no menor de los episodios detectados, la inflación comienza a descender con posterioridad al episodio, aunque esto resulta más apreciable y frecuente en el caso de los países avanzados que en los emergentes. 
El resultado básico anterior puede subestimar el efecto del shock de precios relativos sobre la tasa de inflación. Cuando se consideran solo aquellos casos que toman como moneda de referencia al USD, el efecto del cambio en el TCR sobre la inflación aumenta significativamente.

Utilizando la información provista por la clasificación de Shambaugh (2004), podemos distinguir entre aquellos casos que administraban el TCN con relación al USD y aquellos que tenían un régimen cambiario con relación a una moneda base distinta del dólar (panel d) ${ }^{7}$. Estos casos presentan una clara asimetría en la respuesta de la tasa de inflación en comparación con los casos donde la referencia cambiaria es otra moneda.

Cabe destacar la diferencia que se observa en la dinámica entre diversas regiones (figura A1 del apéndice online), donde se distingue Latinoamérica y el este de Asia y el Pacífico por la magnitud de la respuesta inflacionaria (al alza) con respecto a Europa y África subsahariana. Al mismo tiempo, se evidencia una clara asimetría en la distribución temporal para estos casos. En su gran mayoría, los episodios están asociados a un aumento en la tasa de inflación y prácticamente no hay casos de desinflación. Esto es particularmente notorio en el caso de Latinoamérica.

Esto en parte refleja lo discutido anteriormente. En el caso de Latinoamérica, el dólar es una moneda de referencia ampliamente difundida, a diferencia del caso europeo o el africano donde se observan muchos casos de fijaciones contra monedas como el marco alemán o el franco. No obstante, es difícil pensar que esta es la única razón fuente de diferencias. La región también se destaca por la baja proporción de episodios de devaluación con desaceleración inflacionaria que presenta. Un hecho que distingue esta región es que, en promedio, presenta las tasas de inflación más elevadas al inicio de los episodios: $292 \%$ anual comparado con un promedio de $11 \%$ para el resto de los episodios.

\footnotetext{
7 El criterio de Shambaugh se basa en el análisis del comportamiento de un conjunto de TCN bilaterales. En Shambaugh (2004), la moneda de referencia surge de analizar si las economías fijan el TCN (mantienen una banda de $+/-2 \%$ ) contra el USD, o monedas de relevancia dentro de una región. En los casos donde no se detecta un régimen de fijación, la moneda base se asigna en función del análisis histórico. En caso de que ninguno de estos criterios fuera claro, se asume que la moneda de referencia es el USD.
} 
Figura 2. Comportamiento de la inflación durante los episodios de devaluación

(a) TOTAL (562 episodios)

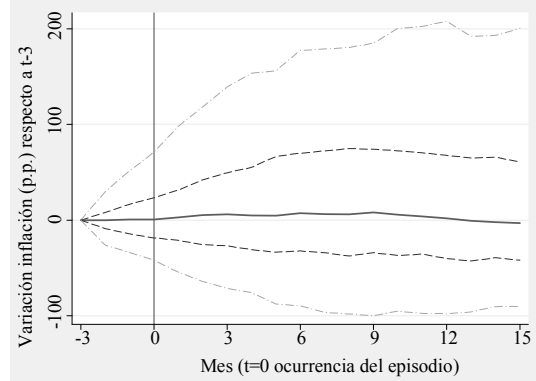

(c) EMERGENTES (371 episodios)

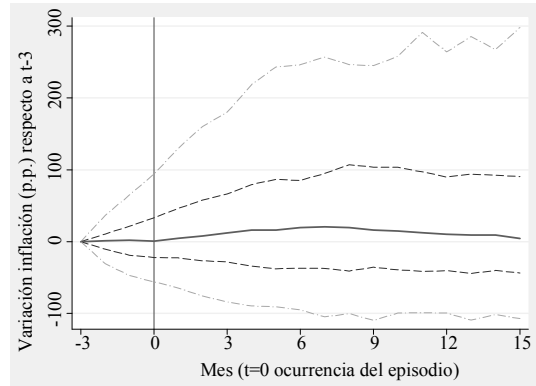

(b) AVANZADOS (191 episodios)

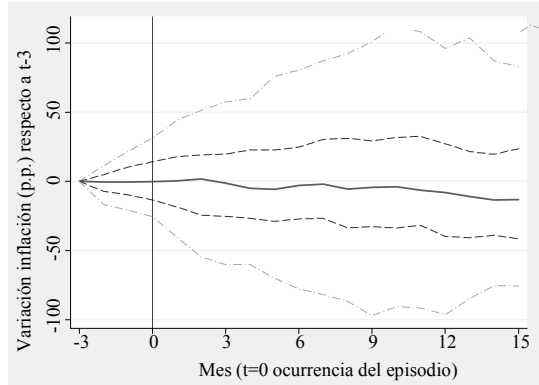

(d) USD COMO MONEDA DE REFERENCIA

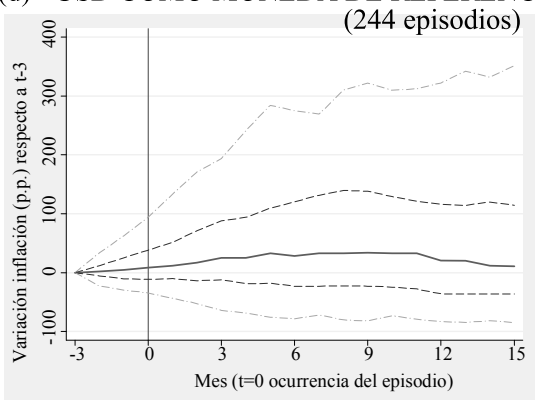

Nota: los gráficos muestran la distribución temporal de la variación (acumulada) de la inflación en los episodios detectados bajo $\psi=0.1$ (571 episodios). La línea roja muestra la mediana, las azules punteadas los percentiles 25 y 75 y las grises los percentiles 90 y 10, respectivamente. La variación en la inflación se mide como el cambio, en puntos porcentuales, con respecto a (t-3). Se excluyen los episodios para los que no se cuenta con datos de inflación en los 6 meses previos al comienzo del episodio. Fuente: elaboración propia sobre la base de IFS (FMI).

II.4. Clasificación de episodios "aceleradores" y "no aceleradores"

Una vez identificados los episodios de depreciación real, se clasifica a los mismos en función de los cambios observados en la tasa de inflación (acumulada 12 meses). Se evalúa el cambio en esta tasa de inflación a $\mathrm{H}_{\pi}$ meses del episodio a efectos de distinguir los casos donde se advierten aceleraciones apreciables (episodios "aceleradores") de los que no ("no aceleradores"). Se destaca que $\mathrm{H}_{\pi}$ no necesariamente coincide con el horizonte $\mathrm{H}_{q}$ mencionado antes. 
Un episodio devaluatorio es considerado "acelerador" cuando se cumplen, de forma concomitante, dos condiciones:

1. Transcurrido el horizonte la tasa de inflación (interanual) exhibe un incremento de al menos veces con respecto a la tasa de inflación anual al comienzo del episodio ( $\mathrm{t}-3)$ :

$$
\pi_{t+\mathrm{H}_{\pi}}>\gamma \pi_{t-3} \operatorname{con} \gamma>1
$$

2. La inflación aumenta en al menos puntos porcentuales. Esto es, se fija una condición de cambio absoluto en la tasa interanual, de la siguiente forma:

$$
\pi_{t+\mathrm{H}_{\pi}}-\pi_{t-3}>\varepsilon
$$

A efectos de simplificar la cantidad de parámetros "libres", optamos por una regla que define en función del parámetro de forma tal que la condición de cambio absoluta incremente junto con la condición porcentual:

$$
\varepsilon=\frac{(\gamma-1)}{10}+0.01
$$

Bajo ambas condiciones, los episodios de aceleración son aquellos que exhiben (i) un incremento proporcional significativo en la tasa de inflación y, como consecuencia de este incremento, (ii) un cambio absoluto no despreciable. Esto es particularmente relevante para los casos de inflación "baja" (menor al $10 \%$ anual), donde la restricción operativa para identificar casos de aceleración es, seguramente, (5) en lugar de (4).

\section{II.5. Características de los episodios "aceleradores"}

En la tabla 2 se muestra la proporción o frecuencia de los episodios aceleradores para distintas combinaciones de (i) la intensidad del cambio en (i.e. el valor del umbral $\psi$ ) y (ii) el valor mínimo de cambio "significativo" en la tasa de inflación.

Se aprecia que cuanto más restrictivo el parámetro de identificación $\psi$ (mayor la variación mínima exigida al TCR), más elevada es la frecuencia de episodios aceleradores, dado un umbral $\gamma$ de cambio en la tasa de inflación. Esto nos permite entrever un primer resultado central, la ocurrencia de un episodio 
"acelerador" está asociado a la magnitud del shock devaluatorio. Cuanto mayor es el shock, más elevada la frecuencia.

A medida que se extiende el horizonte de evaluación para los cambios en la inflación $-H_{\pi}$ - la proporción decrece, lo que marcaría la importancia de los factores de segunda ronda: procesados los impactos de primera ronda, el proceso se sostiene solo sobre la base de los segundos.

Tabla 2. Frecuencia de episodios "aceleradores" - sensibilidad a intensidad de cambios en $q(\psi)$ y umbrales de incremento en la inflación $(\gamma)-H_{\pi}=12$ meses

\begin{tabular}{|c|c|c|c|c|c|c|}
\hline & \multicolumn{6}{|c|}{ Variación trimestral mínima acumulada en $-=$} \\
\hline \multirow{7}{*}{$\begin{array}{l}\text { Cambio mínimo } \\
\text { en con respecto a }\end{array}$} & & 0.05 & 0.10 & 0.15 & 0.20 & 0.25 \\
\hline & 1.05 & $36,9 \%$ & $46,7 \%$ & $53,8 \%$ & $60,1 \%$ & $68,3 \%$ \\
\hline & 1.10 & $32,4 \%$ & $42,9 \%$ & $51,5 \%$ & $59,4 \%$ & $67,3 \%$ \\
\hline & 1.15 & $29,4 \%$ & $38,6 \%$ & $48,9 \%$ & $56,5 \%$ & $64,4 \%$ \\
\hline & 1.20 & $26,8 \%$ & $36,3 \%$ & $46,2 \%$ & $53,6 \%$ & $62,5 \%$ \\
\hline & 1.25 & $23,4 \%$ & $32,3 \%$ & $44,0 \%$ & $52,9 \%$ & $60,6 \%$ \\
\hline & 1.30 & $21,4 \%$ & $29,5 \%$ & $41,0 \%$ & $52,2 \%$ & $58,7 \%$ \\
\hline
\end{tabular}

Fuente: elaboración propia sobre la base del IFS (FMI).

Queda claro que, si bien no es un fenómeno de elevada frecuencia para todas las combinaciones posibles de parámetros, la incidencia no es para nada despreciable. Si consideramos los episodios con umbrales mínimos de devaluación real de entre $10 \%$ y $15 \%$ observamos que, a 12 meses de la ocurrencia del mismo, un 51.5-36.3\% de los eventos muestra tasas de inflación anuales superiores en un $10-20 \%$ (2-3 p. p. de cambio absoluto mínimo) a las tasas que prevalecían antes de la devaluación.

Para lo que resta del trabajo, nos focalizamos en los eventos de incremento del TCR con un piso del $10 \%(\psi=0.1)$, por presentar un número de eventos significativo.

Asimismo, se opta por centrarse en horizontes de evaluación del cambio en la tasa de inflación $\left(H_{\pi}\right)$ de 6 y 12 meses. Esto, además de permitir simplificar la exposición, obedece a dos motivos. Por un lado, se considera que estos horizontes son de una longitud suficiente para que se manifiesten los efectos de primera y 
segunda ronda, así como las respuestas de política. Por otra parte, en el caso de horizontes más extensos, resulta muy difícil descartar la influencia de otros factores en la tasa de inflación, no relacionados con el shock devaluatorio inicial al comienzo del episodio.

La figura 3 muestra la distribución temporal de las 2 variables centrales - el TCR y la tasa de inflación- en torno a los dos tipos de episodios clasificados. En función de lo comentado anteriormente, se analizan los episodios bajo el horizonte $H_{\pi}=12 \mathrm{~m}$. En el apéndice, el gráfico A2 muestra la distribución de la dinámica de ambas variables para las regiones donde se concentran los episodios de devaluación real.

La varianza de ambos tipos de episodios es muy diferente, tanto para el TCR como para la tasa de inflación. La distribución de la serie de tiempo está dominada por lo que ocurre con las economías emergentes; no hay diferencias apreciables entre la distribución de estas economías y la muestra completa (paneles a y b). La magnitud del shock (mediano) del TCR en el caso de los episodios "aceleradores" es claramente mayor que en los "no aceleradores", con una tendencia a continuar aumentando en los meses posteriores a ocurrido el episodio.

Se aprecia que la inflación en el caso de los eventos aceleradores aumenta a lo largo del horizonte de evaluación seleccionado (12 meses), aunque comienza a bajar con posterioridad, pero manteniéndose en niveles superiores a la dinámica previa. Dentro de los episodios "no aceleradores", una parte de la distribución muestra aceleraciones en horizontes inferiores; empero, se observa que la inflación disminuye por debajo del umbral - $\gamma$ - a una velocidad elevada y por tanto son clasificados como "no aceleradores".

Cuando consideramos la distribución de los episodios en países que referencian su moneda al dólar, se aprecia que el shock inicial al TCR es algo mayor. Asimismo, la evolución de la inflación es semejante a la distribución de los países emergentes, para ambos tipos de episodios, si bien se aprecia que la intensidad en la aceleración es algo mayor.

En la tabla A2 del apéndice se presenta la distribución de la frecuencia de episodios "aceleradores" en función de los distintos períodos (décadas), regímenes cambiarios, regiones geográficas y nivel de desarrollo de los países que componen la muestra, para los parámetros seleccionados. 
Figura 3. Evolución del TCR y la tasa de inflación en los episodios "aceleradores" y "no aceleradores"

(a) Muestra completa - 562 episodios (desarrollados y emergentes)
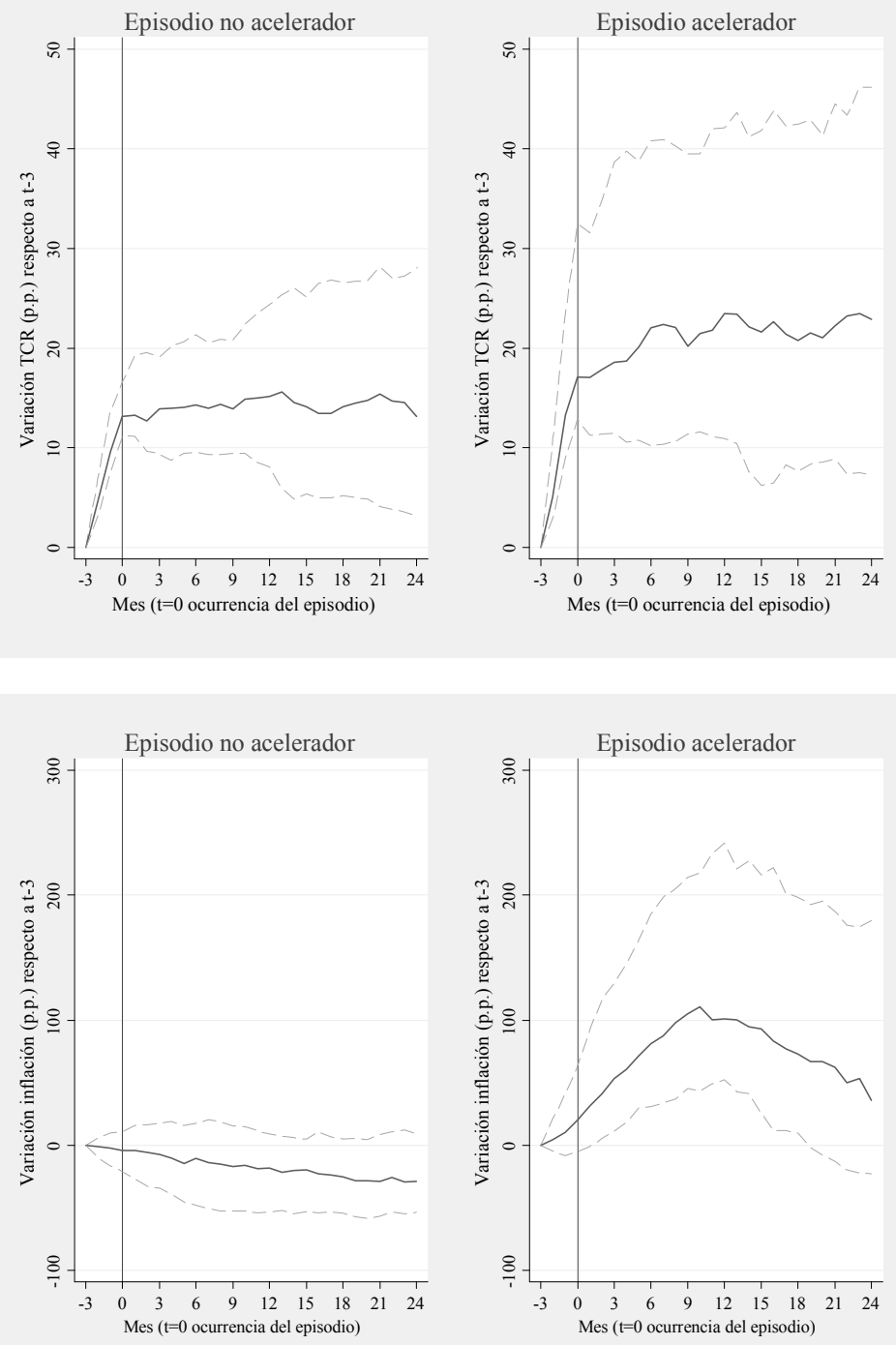
(b) Solamente episodios ocurridos en países emergentes - 371 episodios
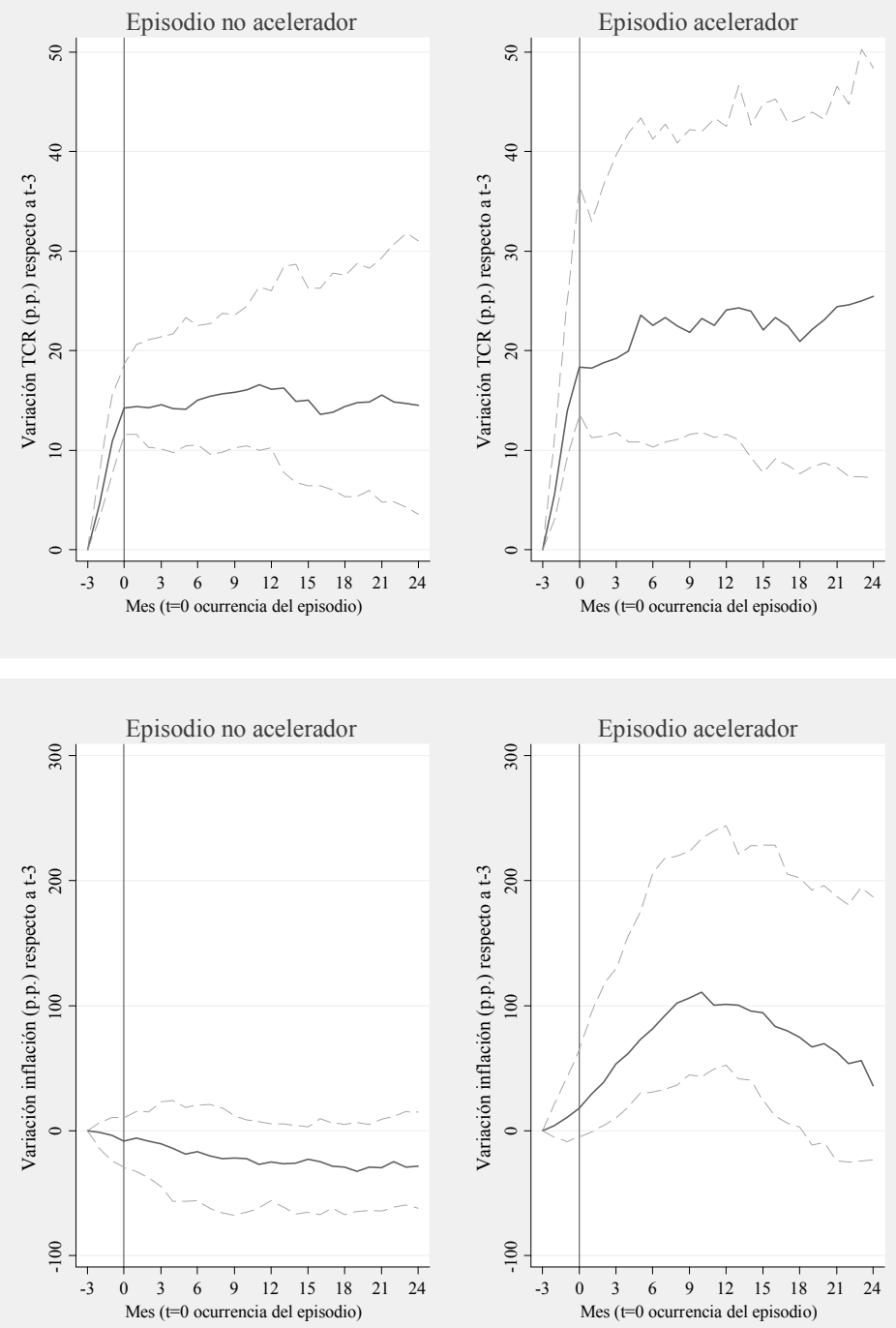

Nota: los gráficos muestran la distribución temporal de la variación del TCR y la inflación en torno a los episodios detectados bajo, y horizonte . La línea roja muestra la mediana, mientras que las grises punteadas representan los percentiles 25 y 75 . La variación del TCR y la inflación se miden como el cambio acumulado, en puntos porcentuales, de ambas variables con respecto al valor observado en ( $\mathrm{t}-3)$.

Fuente: elaboración propia sobre la base del IFS (FMI). 
No se evidencia un cambio en la frecuencia de los casos "aceleradores" a lo largo del tiempo: mientras en las décadas del 70 y el 80 , el $34 \%$ de los casos constituían episodios "aceleradores", en la década del 2000 solo el $28 \%$ de los casos muestra cambios prolongados en la inflación. Esto es más notorio cuando restringimos el análisis al caso de países con referencia al USD.

La frecuencia media difiere sensiblemente para las economías desarrolladas y emergentes y, de forma semejante, se observa una marcada disparidad entre las regiones: Latinoamérica y África sub-sahariana exhiben la mayor frecuencia de episodios aceleradores. Esta diferencia de frecuencias tiene asociada también una diferencia significativa en la dinámica del TCR y la inflación en torno al evento. El gráfico A1 del apéndice muestra el comportamiento disímil que presentan las regiones de mayor frecuencia con respecto a dos regiones donde la incidencia es menor (Europa y el este de Asia y el Pacífico).

La tabla 3 muestra la relación entre el régimen inflacionario previo al episodio devaluatorio y la frecuencia de los episodios "aceleradores", tanto para emergentes como para desarrollados. Mayores niveles de inflación previos a la ocurrencia del episodio devaluatorio se asocian, ceteris paribus, a una mayor frecuencia de eventos de aceleración inflacionaria.

La proporción de casos aceleradores se incrementa en los episodios de mayor inflación inicial. La relación emerge más nítidamente cuando consideramos a la muestra en su conjunto y no tenemos en cuenta el grado de desarrollo, pero esto podría reflejar una menor incidencia de los efectos de primera ronda en los países desarrollados (mayor presencia de pricing-to-market) en lugar de una diferencia en los efectos de segunda ronda y el funcionamiento del mercado de trabajo.

La relación entre inflación inicial y la frecuencia de un episodio "acelerador" puede ser producto de, al menos, dos causas no necesariamente excluyentes.

A nivel macroeconómico, puede ser explicado por el desarrollo de mecanismos indexatorios en entornos de elevada inflación (Dornbusch y Fischer, 1993). Desde un punto de vista "microeconómico", los entornos de elevada inflación y los shocks con consecuencias inflacionarias tienden a "depreciar" la información de precios relativos en manos de los consumidores, lo que tiende a incrementar el poder de monopolio de las firmas si la búsqueda de información es costosa (Tommasi, 1994). En consecuencia, ante un shock de costos las firmas tienden a incrementar su mark-up exacerbando la respuesta inflacionaria. 
Tabla 3. Episodios "aceleradores" y entorno inflacionario

Episodios identificados bajo $\psi=0.1, \gamma=1.1$

\begin{tabular}{lccc}
\hline $\begin{array}{c}\text { Inflación anual en los } 6 \text { meses } \\
\text { previos al episodio }\end{array}$ & & & $\begin{array}{c}\text { Cant. de } \\
\text { episodios }\end{array}$ \\
\hline Avanzados & $14 \%$ & $14 \%$ & 191 \\
\hline Menor a $10 \%$ & $11 \%$ & $12 \%$ & 140 \\
$10-30 \%$ & $17 \%$ & $13 \%$ & 47 \\
$30-50 \%$ & $100 \%$ & $100 \%$ & 1 \\
$50-100 \%$ & - & - & 0 \\
\hline Mayor a $100 \%$ & $67 \%$ & $67 \%$ & 3 \\
\hline Emergentes & $51 \%$ & $52 \%$ & 371 \\
\hline Menor a $10 \%$ & $50 \%$ & $55 \%$ & 178 \\
$10-30 \%$ & $43 \%$ & $48 \%$ & 128 \\
$30-50 \%$ & $77 \%$ & $54 \%$ & 26 \\
$50-100 \%$ & $65 \%$ & $50 \%$ & 20 \\
\hline Mayor a $100 \%$ & $68 \%$ & $42 \%$ & 19 \\
\hline Total general & $38 \%$ & $39 \%$ & 562 \\
\hline
\end{tabular}

Fuente: elaboración propia sobre la base del IFS (FMI).

Un rasgo a destacar es la relativamente baja frecuencia de eventos aceleradores en el caso de economías que operaban con tasas de inflación anual superior al $100 \%$, en especial cuando consideramos un horizonte -- superior a los 6 meses. Lo que ocurre en la mayoría de los casos es que la devaluación es el disparador de un evento de hiperinflación que, como señala la experiencia histórica, son fenómenos de corta duración en el tiempo.

La tabla A3 del apéndice representa la relación entre el estado del nivel de actividad y la ocurrencia de los episodios aceleradores. Se muestra la distribución anual de la brecha de producto (reportando los percentiles 25, 50 y 75). No se aprecian grandes diferencias entre las distribuciones para cada tipo de episodio, por lo que es de esperar que el estado del nivel de actividad no contribuya significativamente para explicar la ocurrencia del fenómeno de interés. 
Tabla 4. Influencia de la política monetaria ${ }^{8}$

Frecuencia de episodios "aceleradores" identificados bajo $\psi=0.1$

\begin{tabular}{lcccc}
\hline & \multicolumn{2}{c}{ Avanzados } \\
\cline { 2 - 5 } $\begin{array}{l}\text { Régimen de PM } \\
\text { (año anterior al ep) }\end{array}$ & 6 meses & 12 meses & 6 meses & 12 meses \\
\cline { 3 - 5 } & $7 \%$ & $7 \%$ & $16 \%$ & $16 \%$ \\
\hline IT & $9 \%$ & $9 \%$ & $46 \%$ & $46 \%$ \\
\hline Resto & $7 \%$ & \multicolumn{3}{c}{$H_{\pi}=$} \\
\hline
\end{tabular}

Nota: clasificación de episodios con regímenes de IT construida sobre la base de trabajos identificados en la tabla A1 del apéndice.

Fuente: Elaboración propia en base a IFS (FMI)

Finalmente, cabe destacar la influencia aparente del régimen de política monetaria y de la estrategia cambiaria adoptada. En la tabla 4 se muestra la frecuencia de episodios aceleradores en función del régimen de política monetaria del Banco Central en el año previo a la ocurrencia del evento. Se observa un cambio drástico en la frecuencia para aquellos eventos donde el Banco Central sigue un régimen de IT, en especial para el caso de las economías emergentes.

Este resultado es razonable. Bajo IT esperamos que la autoridad monetaria reaccione más prontamente y con mayor vigor ante incrementos en la inflación. No obstante, debe tenerse presente que los regímenes de IT se han establecido en economías que partían de tasas bajas de inflación. El efecto asociado al régimen de IT podría en este sentido ser (i) consecuencia de un entorno de bajas tasas de inflación, o (ii) reflejar la mejor calidad de las instituciones de política asociados a este régimen de política monetaria.

Una respuesta preliminar en favor de la segunda hipótesis surge de la tabla 5. Allí se muestran la cantidad de episodios "aceleradores" y "no aceleradores" que han ocurrido para países emergentes a partir del año 1990 con inflaciones iniciales bajas (inferior al $10 \%$ anual), distinguiendo entre los casos donde el banco central tenía en funcionamiento un régimen de IT u otro régimen. Se desprende que, aun bajo condiciones de baja inflación, la incidencia de los episodios "aceleradores" es sustancialmente menor bajo regímenes de IT que bajo otros esquemas de política monetaria.

8 A efecto de hacer comparable los regímenes de política monetaria, la tabla se refiere a la frecuencia de episodios aceleradores solo para las décadas posteriores a 1990, dado que no existen casos de IT con anterioridad. Véase Hammond (2012). 
Tabla 5. Cantidad de episodios "aceleradores" y "no aceleradores" bajo IT Países emergentes, 1990-2014, inflación inicial "baja" (< al 10 \%)

\begin{tabular}{lcccc}
\hline \multirow{2}{*}{$\begin{array}{c}\text { Régimen de PM } \\
\text { (año anterior al } \\
\text { episodio) }\end{array}$} & $H_{\pi}=6$ meses & \multicolumn{4}{c}{$H_{\pi}=12$ meses } \\
\cline { 2 - 5 } & No acelerador & Acelerador & No acelerador & Acelerador \\
\hline$I T$ & 20 & 3 & 19 & 4 \\
Resto & 70 & 36 & 61 & 45 \\
\hline
\end{tabular}

Fuente: elaboración propia sobre la base del IFS (FMI).

En cuanto a la influencia de la política cambiaria, la tabla 6 muestra el comportamiento del anclaje cambiario en cada tipo de episodio. El grado de anclaje cambiario se define como la variación en el TCN en los $H_{\pi}$ meses posteriores a lo ocurrencia del shock en el TCR, con respecto a la variación que había exhibido en un período de igual longitud antes de dicho shock. Formalmente:

$$
A C_{H_{\pi}}=\frac{T C N_{t+H_{\pi}} / T C N_{t}}{T C N_{t-3} / T C N_{t-3-H_{\pi}}}-1
$$

Podemos ver que cuando tiende a cero o, incluso, es negativo, mayor es la influencia desinflacionaria del TCN (mayor es su rol como ancla). En el caso donde es equivalente a cero, la devaluación es esencialmente un cambio de la paridad en el marco de un régimen cambiario fijo.

Tabla 6. Influencia de la política cambiaria Grado de anclaje en episodios "aceleradores" y "no aceleradores". Promedio de anclaje por tipo de episodio.

\begin{tabular}{ccccc}
\hline & \multicolumn{2}{c}{ Avanzados } & \multicolumn{2}{c}{ Emergentes } \\
\cline { 2 - 5 } Tipo de episodio & \multicolumn{3}{c}{$H_{\pi}=$} \\
\cline { 2 - 5 } & 0.03 & 0.04 & 0.03 & 0.09 \\
\hline "No acelerador" & 0.08 & 0.33 & 0.34 & 0.62 \\
\hline
\end{tabular}

Fuente: elaboración propia sobre la base del IFS (FMI). 
Se aprecia que los episodios "aceleradores" se corresponden con menores niveles de anclaje cambiario. Esto es más notorio en el caso de los emergentes.

El hecho estilizado que surge es que, en promedio, los episodios "no aceleradores" se distinguen por presentar un TCN que, luego de ocurrida la devaluación, tiende a fijarse, o bien mantienen una tasa de devaluación semejante a la que prevaleció previamente a la ocurrencia del episodio devaluatorio. Por ejemplo, en una devaluación en el marco de un régimen de tipo de cambio fijo los episodios "no aceleradores" se asocian a cambios discretos en el TCN (una especie de cambio "once and for all"). Por oposición, los episodios "aceleradores" se asocian a casos donde el TCN se deprecia a una tasa más alta de la que exhibía antes del cambio en el TCR.

\section{ANÁLISIS ECONOMÉTRICO: PROBABILIDAD DE EPISODIOS "ACELERADORES"}

\section{III.1. Estrategia de estimación}

Pasamos a analizar los determinantes de la frecuencia de episodios aceleradores teniendo en cuenta los rasgos estilizados discutidos. Los episodios detectados y clasificados en el panel de frecuencia mensual son agregados en una base de corte transversal junto con variables disponibles bajo una frecuencia anual (e. $g$. la brecha de producto y el régimen de política monetaria). La estrategia de análisis propuesta presenta de esta forma un carácter "episódico".

La clasificación de los episodios entre "aceleradores" y "no aceleradores" nos permite realizar un análisis mediante modelos de probabilidad (un probit). Esto es, la variable dependiente de interés () adquiere un valor igual a 1 si el episodio, bajo un horizonte y un valor específico del parámetro, es clasificado como "acelerador", y 0 en caso contrario.

Siguiendo el análisis de las secciones anteriores, se utiliza una especificación dada por la siguiente combinación de parámetros: , , y meses, lo que implica un total de 571 episodios. Bajo esta especificación, se analiza la probabilidad de ocurrencia de episodios "aceleradores" clasificados bajo y horizontes -- de 6 y 12 meses. De esta forma, se clasifican 183 y 142 episodios como "aceleradores" para horizontes a 6 y 12 meses, respectivamente. No obstante, a efectos de considerar 
potenciales problemas de medición y clasificación, se estiman y reportan los resultados para diversas combinaciones de los parámetros ${ }^{9}$.

El modelo de probabilidad condicional analiza la incidencia de los siguientes factores: (i) la tasa de inflación inicial, (ii) la presencia o ausencia de regímenes monetarios tipo IT, (iii) el grado de "anclaje cambiario" posterior a la ocurrencia del shock devaluatorio, y (iv) las condiciones de demanda efectiva. En términos formales, el modelo de probabilidad a estimar es del siguiente estilo ${ }^{10}$ :

$$
P(\chi=1 \mid X)=G\left(\theta_{0}+\theta_{1} \pi_{t-1}+\theta_{2} I T+\theta_{3} A C+\theta_{4} G A P+d_{T}\right)
$$

Donde $\pi_{t-1}$ es la tasa de inflación al inicio del episodio, es una variable dummy que toma valores igual a uno si hay un régimen de inflation-targeting, indica el grado de anclaje cambiario para el horizonte $H_{\pi}, G A P$ es una variable que representa la brecha de producto (exceso de demanda), y, finalmente, $d_{T}$ son un conjunto de variables binarias por década utilizadas como controles de efectos temporales.

Usamos la tasa de inflación pasada para analizar, indirectamente, la contribución del entorno inflacionario en la ocurrencia de un episodio "acelerador". La variable GAP intenta captar los efectos asociados al desempleo y su influencia en la evolución de los salarios. Finalmente, las variables asociadas al comportamiento del TCN y el régimen monetario permiten apreciar la influencia del régimen y la efectividad de las respuestas de política económica (monetarias y/o cambiarias) que aplican las autoridades.

Como establecen Horrace y Oaxaca (2006), excepto bajo supuestos muy restrictivos los modelos lineales de probabilidad generan estimaciones sesgadas e inconsistentes de los parámetros de interés. Si bien los modelos no lineales presentan sesgos en muestras pequeñas, al menos son consistentes. Asimismo, en caso de errores de medición en la variable dependiente, las implicancias son menos severas para los modelos no lineales con respecto a los lineales: en estos últimos, los parámetros son simplemente no identificables (Hausman, Abrevaya y Scott-Morton, 1998).

9 Por razones de espacio estos resultados no se reportan en este trabajo. El autor los pone a disposición para quien los requiera.

10 La función $\mathrm{G}$ representa una función de distribución acumulada definida sobre el índice definido por los parámetros y variables explicativas $(\theta X)$. En el caso de los modelos probit, la función $\mathrm{G}$ es la función asociada a la normal estándar. La transformación a partir de la función G(.) implica que los efectos parciales no sean constantes y dependan del punto del índice donde se calcule (Wooldridge, 2010). 
Un control importante presente en las estimaciones es la magnitud del shock devaluatorio. Los episodios con aceleración inflacionaria podrían deberse simplemente a un mayor cambio en el TCR. Todas las estimaciones controlan por la tasa de variación del TCR acumulada en los 3 meses en los que se evalúa la ocurrencia del episodio.

Se opta por descartar episodios extremos que por sus características intrínsecas no se sostienen en el tiempo, afectando la estabilidad y significatividad de los parámetros de interés. En las estimaciones se restringe la muestra a aquellos eventos donde la tasa de inflación anual promedio de los 6 meses previos al episodio es inferior al $100 \%{ }^{11}$. Este procedimiento es semejante al utilizado por Ca'Zorzi, Hahn y Sánchez (2007).

\section{III.2. Resultados}

Las tablas 7 a 9 presentan los resultados para horizontes de cambio en la inflación $-H_{\pi}$ - de 6 y 12 meses. En todas las tablas se reportan los efectos marginales valuados en los valores medios de los regresores.

La tabla 7 analiza los determinantes de la frecuencia de episodios "aceleradores", considerando el total de casos, es decir, agrupando casos de países avanzados y emergentes. Las columnas (1) a (3) se refieren a episodios clasificados teniendo en cuenta horizontes de 6 meses, mientras que las columnas (4) a (6) refieren a horizontes de 12 meses. Por su parte, las columnas (1) y (4) muestran los resultados de regresiones para todo el período (1970-2014), mientras que las columnas (2) a (3) y (5) a (6) descomponen la muestra en 2 subperíodos: 1970-1989 y 1990-2014. De esta manera, evaluamos la estabilidad de los coeficientes a diferentes entornos temporales, como una estrategia de control diferente del uso de las variables dummy por década (identificadas como [1990-1999] y [2000-2014]).

Se aprecia que una mayor tasa de inflación inicial incrementa significativamente la probabilidad de ocurrencia de un episodio acelerador. Cada punto de inflación anual implica un incremento aproximado de 0,8 \%-0,4 \% en la probabilidad de ocurrencia. El resultado se mantiene para ambos horizontes y para los distintos subperíodos. Asimismo, es robusta a variaciones en los parámetros $\psi$ y $\gamma$ utilizados en la identificación (resultados no reportados).

11 Si bien este umbral está lejos de la definición clásica de hiperinflaciones de Cagan (1956), coincide con el límite utilizado para distinguir los eventos de inflación extrema o hiperinflaciones "modernas" (Fischer, Sahay y Végh, 2002). 


\section{Tabla 7. Probabilidad de episodios aceleradores: todos los países Estimaciones probit.}

Episodios identificados y clasificados bajo $\psi=0.1$, y $\gamma=1.2$

\begin{tabular}{|c|c|c|c|c|c|c|}
\hline \multirow[b]{2}{*}{ VARIABLES } & \multicolumn{3}{|c|}{$=1$ si episodio acelerador $\left(H_{\pi}=6\right.$ meses $)$} & \multicolumn{3}{|c|}{$=1 \mathrm{si}$ episodio acelerador $\left(\mathrm{H}_{\pi}=12\right.$ meses $)$} \\
\hline & $\begin{array}{c}(1) \\
\text { Caso Base }\end{array}$ & $\begin{array}{c}(2) \\
1970-1989\end{array}$ & $\begin{array}{c}(3) \\
1990-2014\end{array}$ & $\begin{array}{c}(4) \\
\text { Caso Base }\end{array}$ & $\begin{array}{c}(5) \\
1970-1989\end{array}$ & $\begin{array}{c}(6) \\
1990-2014\end{array}$ \\
\hline Inflacion anual $(12 \mathrm{~m})$ en $\mathrm{t}-1$ & $\begin{array}{c}0.719^{* * *} \\
{[0.15218]}\end{array}$ & $\begin{array}{c}0.565 * * * \\
{[0.14921]}\end{array}$ & $\begin{array}{c}0.867 * * * \\
{[0.25316]}\end{array}$ & $\begin{array}{c}0.400 * * * \\
{[0.12323]}\end{array}$ & $\begin{array}{c}0.378 * * * \\
{[0.14215]}\end{array}$ & $\begin{array}{c}0.436^{*} \\
{[0.23938]}\end{array}$ \\
\hline Output gap en T-1 & $\begin{array}{c}-1.591^{*} \\
{[0.88452]}\end{array}$ & $\begin{array}{c}-0.440 \\
{[1.20614]}\end{array}$ & $\begin{array}{c}-2.509^{*} \\
{[1.38021]}\end{array}$ & $\begin{array}{c}-0.188 \\
{[0.83957]}\end{array}$ & $\begin{array}{c}0.468 \\
{[1.12702]}\end{array}$ & $\begin{array}{c}0.432 \\
{[1.33812]}\end{array}$ \\
\hline Output gap en $\mathrm{T}$ & $\begin{array}{c}-0.061 \\
{[0.72933]}\end{array}$ & $\begin{array}{c}-1.787 \\
{[1.17715]}\end{array}$ & $\begin{array}{c}1.007 \\
{[0.92860]}\end{array}$ & $\begin{array}{c}0.948 \\
{[0.75548]}\end{array}$ & $\begin{array}{c}0.063 \\
{[1.10657]}\end{array}$ & $\begin{array}{c}1.762 * \\
{[1.04412]}\end{array}$ \\
\hline$=1$ si Inflation Targeting en T-1 & $\begin{array}{l}-0.217 * * * \\
{[0.05934]}\end{array}$ & & $\begin{array}{c}-0.163 * * \\
{[0.06481]}\end{array}$ & $\begin{array}{l}-0.232 * * * \\
{[0.05541]}\end{array}$ & & $\begin{array}{c}-0.170^{* *} \\
{[0.06706]}\end{array}$ \\
\hline Anclaje cambiario $6 \mathrm{~m}^{1}$ & $\begin{array}{c}-0.223^{*} \\
{[0.12146]}\end{array}$ & $\begin{array}{c}-0.307 \\
{[0.19302]}\end{array}$ & $\begin{array}{c}-0.159 \\
{[0.13923]}\end{array}$ & & & \\
\hline Anclaje cambiario $12 \mathrm{~m}^{1}$ & & & & $\begin{array}{c}-0.098 * * \\
{[0.04812]}\end{array}$ & $\begin{array}{c}-0.110^{*} \\
{[0.05869]}\end{array}$ & $\begin{array}{l}-0.454 * * * \\
{[0.10915]}\end{array}$ \\
\hline [1990-1999] & $\begin{array}{c}0.009 \\
{[0.05257]}\end{array}$ & & & $\begin{array}{c}0.037 \\
{[0.05189]}\end{array}$ & & \\
\hline$[2000-2014]$ & $\begin{array}{c}0.093 \\
{[0.06967]}\end{array}$ & & & $\begin{array}{c}0.103 \\
{[0.06736]}\end{array}$ & & \\
\hline Shock inicial al TCR $\left(\mathrm{q}_{\mathrm{t}} / \mathrm{q}_{\mathrm{t}-3}\right)$ & $\begin{array}{c}0.362 * \\
{[0.18861]}\end{array}$ & $\begin{array}{c}0.580 * \\
{[0.35254]}\end{array}$ & $\begin{array}{c}0.237 \\
{[0.20026]}\end{array}$ & $\begin{array}{c}-0.093 \\
{[0.07606]}\end{array}$ & $\begin{array}{c}-0.153 \\
{[0.09337]}\end{array}$ & $\begin{array}{c}0.252 \\
{[0.20708]}\end{array}$ \\
\hline $\begin{array}{l}\text { Observaciones } \\
\text { Pseudo R2 }\end{array}$ & $\begin{array}{c}492 \\
0.149\end{array}$ & $\begin{array}{c}241 \\
0.157\end{array}$ & $\begin{array}{c}251 \\
0.162\end{array}$ & $\begin{array}{c}492 \\
0.0630\end{array}$ & $\begin{array}{c}241 \\
0.0484\end{array}$ & $\begin{array}{c}251 \\
0.145\end{array}$ \\
\hline
\end{tabular}

Se reportan los efectos marginales valuados en los valores medios de los regresores. Errores estándar robustos entre corchetes. Se excluyen los episodios con inflación anual promedio superior al $100 \%$ en los 6 meses previos. $* * * \mathrm{p}<0.01, * * \mathrm{p}<0.05, * \mathrm{p}<0.1$.

1/ La variable "anclaje cambiario" se multiplica por (-1) para facilitar la lectura de los resultados.

Fuente: elaboración propia sobre la base del IFS (FMI).

Surge también que la brecha de producto (del año $T$ en que ocurre el episodio, o el año anterior) presenta una influencia moderada o inclusive no significativa a la hora de explicar la frecuencia. En otras palabras, el estado del nivel de actividad parece tener poca influencia en la dinámica de la tasa de inflación para estos horizontes. En algunos casos, el signo es contrario al esperado, con una asociación positiva entre la brecha de producto y la aceleración de la inflación ${ }^{12}$.

12 Recordemos que, en nuestro caso, la brecha se define como la diferencia entre la tendencia HP y el PIB observado. De esta forma, una brecha positiva implica un "exceso de oferta" o una "deficiencia de demanda agregada", lo que debería ejercer una influencia negativa sobre la tasa de inflación. 
Se aprecia que la existencia previa de un régimen de IT implica una disminución significativa en la probabilidad de ocurrencia. Asimismo, la política cambiaria parece ejercer un rol significativo: cuanto mayor es el grado de anclaje cambiario con posterioridad al episodio de devaluación (i. e. más cercano al 0 ), menor es la posibilidad de que se experimente una aceleración sustancial en la tasa de inflación.

Las tablas 8 y A4 (apéndice) repiten el análisis anterior, pero separando los episodios entre aquellos ocurridos en países emergentes y avanzados, respectivamente. En la sección anterior habíamos visto que la frecuencia de los episodios "aceleradores" era sumamente distinta entre ambos grupos, al tiempo que la influencia de algunas variables parecía ser disímil.

Tabla 8. Probabilidad de episodios aceleradores: solo emergentes

Estimaciones probit. Episodios identificados y clasificados bajo $\psi=0.1, y \gamma=1.2$

\begin{tabular}{|c|c|c|c|c|c|c|}
\hline \multirow{3}{*}{ VARIABLES } & \multicolumn{3}{|c|}{$=1$ si episodio acelerador $\left(H_{\pi}=6\right.$ meses $)$} & \multicolumn{3}{|c|}{$=1$ si episodio acelerador $\left(H_{\pi}=12\right.$ meses $)$} \\
\hline & (1) & (2) & (3) & (4) & (5) & (6) \\
\hline & Caso Base & $1970-1989$ & $1990-2014$ & Caso Base & $1970-1989$ & $1990-2014$ \\
\hline \multirow[t]{2}{*}{ Inflacion anual (12m) en t-1 } & $0.466^{* * *}$ & $0.336^{* *}$ & $0.674 * *$ & 0.086 & 0.092 & 0.044 \\
\hline & {$[0.14369]$} & {$[0.14018]$} & {$[0.28621]$} & {$[0.13398]$} & {$[0.15795]$} & {$[0.28552]$} \\
\hline \multirow[t]{2}{*}{ Output gap en T-1 } & $-2.231 * *$ & $-2.286^{*}$ & -2.076 & -0.654 & -1.128 & 0.961 \\
\hline & [1.05341] & [1.33988] & [1.63223] & [1.02370] & [1.35614] & [1.56718] \\
\hline \multirow[t]{2}{*}{ Output gap en T } & -0.327 & -1.709 & 0.706 & 1.292 & 0.541 & 2.191 \\
\hline & [0.83996] & {$[1.26725]$} & [1.13778] & [0.89735] & [1.33207] & [1.38478] \\
\hline \multirow{2}{*}{$=1$ si Inflation Targeting en T-1 } & $-0.274 * *$ & & $-0.242 * *$ & $-0.291 * * *$ & & $-0.280 * * *$ \\
\hline & [0.10714] & & [0.10012] & [0.10088] & & {$[0.10079]$} \\
\hline \multirow[t]{2}{*}{ Anclaje cambiario $6 \mathrm{~m}^{1}$} & $-0.322 * *$ & $-0.478^{*}$ & -0.204 & & & \\
\hline & {$[0.15321]$} & {$[0.25870]$} & {$[0.20158]$} & & & \\
\hline \multirow[t]{2}{*}{ Anclaje cambiario $12 \mathrm{~m}^{1}$} & & & & $-0.129 * * *$ & $-0.176^{* * *}$ & $-0.476^{* * *}$ \\
\hline & & & & {$[0.04624]$} & {$[0.06215]$} & {$[0.13301]$} \\
\hline \multirow[t]{2}{*}{ [1990-1999] } & 0.020 & & & 0.040 & & \\
\hline & {$[0.07359]$} & & & {$[0.07173]$} & & \\
\hline \multirow[t]{2}{*}{ [2000-2014] } & 0.015 & & & -0.005 & & \\
\hline & {$[0.08431]$} & & & {$[0.08205]$} & & \\
\hline \multirow[t]{2}{*}{ Shock inicial al TCR $\left(\mathrm{q}_{\mathrm{t}} / \mathrm{q}_{\mathrm{t}-3}\right)$} & 0.319 & 0.454 & 0.243 & $-0.152 * *$ & $-0.264 * * *$ & 0.260 \\
\hline & {$[0.21135]$} & {$[0.29281]$} & {$[0.26072]$} & {$[0.07338]$} & {$[0.09552]$} & {$[0.27301]$} \\
\hline Observaciones & 304 & 140 & 164 & 30 & 140 & 164 \\
\hline Pseudo R2 & 0.119 & 0.137 & 0.119 & 0.0528 & 0.0529 & 0.122 \\
\hline
\end{tabular}

Se reportan los efectos marginales valuados en los valores medios de los regresores. Errores estándar robustos entre corchetes. Se excluyen los episodios con inflación anual promedio superior al $100 \%$ en los 6 meses previos. ${ }^{* * *} \mathrm{p}<0.01,{ }^{* *} \mathrm{p}<0.05,{ }^{*} \mathrm{p}<0.1$

1/ La variable "anclaje cambiario" se multiplica por (-1) para facilitar la lectura de los resultados. Fuente: elaboración propia sobre la base del IFS (FMI). 
Se observa que, en el caso de países emergentes (tabla 8), el nivel de inflación previo al episodio continúa siendo significativo, aunque no resulta estable entre los 2 horizontes considerados. La influencia de la brecha del producto ofrece conclusiones semejantes, si bien la inestabilidad del coeficiente es mayor, en muchos casos encontramos que la influencia es positiva en lugar de negativa.

A diferencia de las dos anteriores, tanto el régimen de IT como el comportamiento de la política cambiaria expresado en el grado de anclaje cambiario conservan su significatividad. En el caso de emergentes, la implementación de regímenes de IT redunda en menor frecuencia de aceleraciones en la tasa de inflación ante aumentos en el TCR. De forma semejante, en los casos donde el TCN ha experimentado un grado elevado de anclaje esto ha redundado en una menor frecuencia de aceleraciones.

En el caso de los países avanzados (tabla A4), las variables asociadas al régimen de IT y el anclaje del TCN no resultan significativas. Asimismo, la brecha de producto, aun cuando es significativa en muchos casos, presenta el signo contrario al indicado por la teoría. La variable de mayor importancia a la hora de explicar los casos de aceleración es la inflación inicial, aunque el coeficiente no presenta gran estabilidad cuando analizamos los subperíodos que componen la muestra.

En definitiva, es poco lo que podemos explicar de los países avanzados. La baja ocurrencia del fenómeno y la menor cantidad de observaciones comparada con los países emergentes dificulta extraer factores explicativos sistemáticos y genera que aumenten los errores.

Comparando los resultados obtenidos para emergentes y avanzados, se aprecia la importancia disímil del régimen de IT para influir sobre el comportamiento de la tasa de inflación. Este resultado está en línea con literatura previa. Ball y Sheridan (2004), con un enfoque de "diferencias en diferencias", muestran que, dentro de la OECD, la adopción de regímenes de IT no ha redundado en mejores resultados en términos de reducción y estabilización de la tasa de inflación para los países con IT comparados con los demás. Gonçalves y Salles (2008) replican este trabajo extendiéndolo para una muestra de 36 economías emergentes y - a diferencia de Ball y Sheridan (2004) - encuentran que los países que adoptaron IT experimentaron mayores caídas en la inflación y una reducción en la volatilidad del producto.

Finalmente, la tabla 10 muestra los resultados para diversas regiones de interés. Las columnas (1) y (5) presentan estimaciones para los episodios ocurridos en América Latina y el Caribe (Latam), las columnas (2) y (6) para el caso de Europa y Asia Central, las columnas (3) y (7) analizan los países del África subsahariana, mientras que las columnas (4) y (8) se refieren a los países con moneda referenciada al USD. 


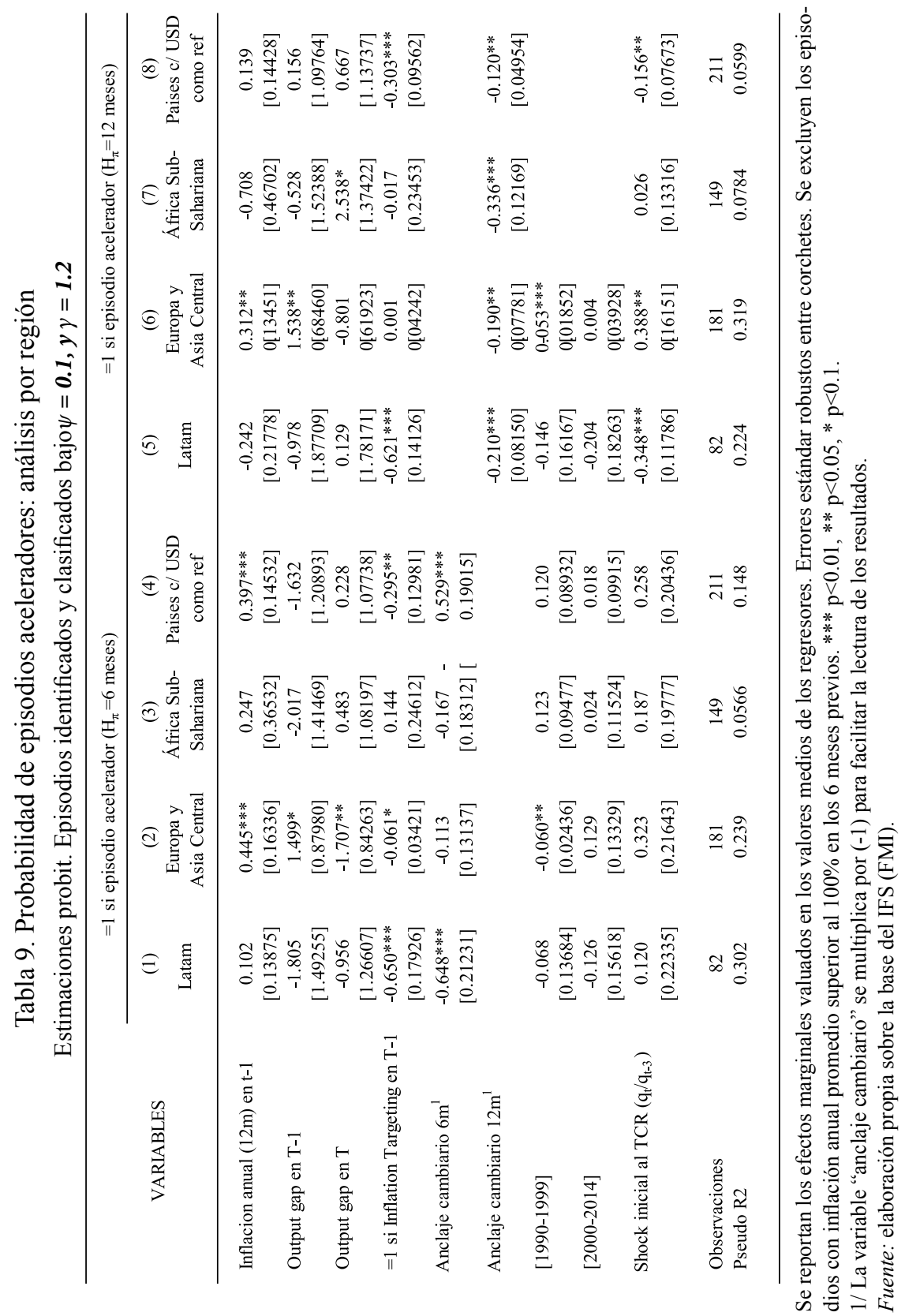


Estos cortes se seleccionaron en función de la disponibilidad de información a efectos de evaluar el grado de homogeneidad del poder explicativo de los diversos factores considerados.

Las regiones presentan diferencias sustanciales en relación con la importancia relativa de las diversas variables para dar cuenta de la frecuencia de episodios "aceleradores". Así, la inflación inicial aparenta ser un factor significativo y robusto para el caso europeo y, en menor medida, para el conjunto de países referenciado al USD. En el caso de Latam, solo cuando consideramos un conjunto de devaluaciones de mayor intensidad, la inflación inicial aparenta ser un factor significativo (resultados no reportados aquí).

Con respecto a la influencia del régimen de política monetaria, se aprecia que esta variable solo resulta significativa y estable para el caso de Latam y los países con referencia cambiaria al USD. De manera semejante, el grado de anclaje cambiario aparenta mayores efectos en estos dos casos.

\section{CONCLUSIONES}

En este trabajo se analizaron empíricamente las condiciones bajo las cuales subas significativas y "duraderas" del TCR desencadenan aceleraciones sustanciales y prolongadas en la tasa de inflación. Se diseñó y aplicó un método de identificación y clasificación para un panel de 98 economías, emergentes y desarrolladas, durante el período 1970-2014. Como resultado se identificaron 571 episodios de devaluación para la especificación elegida. Estos episodios se clasificaron entre "aceleradores" y "no aceleradores" a partir de una regla que evalúa la diferencia (absoluta y porcentual) en la tasa de inflación anual para un horizonte de extensión meses.

Los episodios "no aceleradores" se asocian a dinámicas inflacionarias casi constantes o con una tendencia a la baja y, en menor proporción, a aumentos transitorios de baja intensidad. Por su parte, los "episodios aceleradores" se caracterizan por presentar incrementos significativos en la tasa de inflación, alcanzando un pico dentro de los primeros 12 meses de ocurrido el cambio en el TCR y, posteriormente, exhiben una tendencia a la baja.

La evidencia muestra que, en buena medida, un shock de precios relativos tiende a afectar el nivel general de precios; lo que en versión "dinámica" resulta equivalente a un incremento en la tasa de inflación. Este resultado puede subesti- 
mar el efecto del shock sobre la inflación. Cuando se toman solo casos de países con una historia cambiaria referenciada al USD se observa que la frecuencia de episodios "aceleradores" es significativamente más elevada. Asimismo, la distribución tiende a ser más asimétrica, con un claro sesgo "inflacionario".

Del análisis realizado surge que la ocurrencia de episodios "aceleradores" depende: (i) positivamente de la tasa de inflación inicial, (ii) positivamente de la magnitud del shock cambiario inicial, (iii) negativamente de la existencia previa de un régimen de IT, (iv) negativamente del grado de "anclaje cambiario", y (v) no resulta afectada por el nivel de brecha de producto

Asimismo, se observa una elevada heterogeneidad en la respuesta inflacionaria para las diversas regiones bajo estudio y entre los países "emergentes" y "avanzados".

La presencia de un régimen de IT resulta uno de los determinantes más robustos para influir (negativamente) en la frecuencia de episodios aceleradores, seguido por el grado de anclaje cambiario luego de ocurrido el episodio. La adopción de regímenes de IT parece haber sido un mecanismo efectivo para mantener tasas de inflación bajas y, al mismo tiempo, procesar shocks cambiarios de magnitud significativa evitando aceleraciones inflacionarias persistentes.

La evidencia asociada al nivel de inflación inicial es menos concluyente. No obstante, más allá de que en algunos casos esta variable no resulta significativa, no se observan casos donde la misma presente un signo contrario al esperado.

Se aprecian notables diferencias en la importancia de los diversos factores entre las regiones que componen la muestra de países utilizada. En este sentido, la inflación inicial parece ser un factor determinante en la ocurrencia de aceleraciones inflacionarias al interior de Europa y, en menor medida, en el caso de países referenciados al USD y los de América Latina. Asimismo, la presencia de un régimen de IT resulta significativa al interior de estas últimas dos regiones, pero no se evidencian efectos apreciables al interior de Europa o los países africanos.

La elevada heterogeneidad en la respuesta inflacionaria entre las regiones es un punto interesante que no ha sido debidamente resuelto en el marco de la literatura relevada. En particular, en el caso de América Latina la respuesta de la inflación a episodios devaluatorios es marcadamente diferente al del resto de las regiones consideradas. 
El trabajo permite extraer algunas pequeñas enseñanzas para el diseño de políticas orientadas a morigerar los efectos colaterales de cambios en el TCR. Resulta deseable mantener un entorno de baja inflación combinado con un régimen de política que involucre, al menos, metas explícitas para la evolución futura de la inflación y actúe con velocidad y compromiso para reducir sus desvíos respecto de la meta. Ante la ocurrencia de un cambio en el TCR, es deseable controlar la evolución del TCN a posteriori del shock. Si bien los regímenes de IT suelen prescribirse combinadamente con un régimen de flotación libre, la experiencia de la región ha mostrado que estos pueden convivir con estrategias orientadas a limitar las fluctuaciones del TCN (Céspedes, Chang y Velasco, 2014).

Cabe aclarar que esto no significa que la introducción de un régimen de IT ante la ocurrencia de un shock devaluatorio permita evitar la aceleración inflacionaria. En los casos donde se observa que la inflación no acelera, los regímenes de IT estaban consolidados y la economía había funcionado en entornos de baja inflación durante un largo tiempo. De hecho, es posible que un régimen de IT no reporte beneficios especiales comparados con una política monetaria ejecutada por un Banco Central que busca generar y cuidar su reputación en el control de la inflación.

\section{REFERENCIAS}

Agénor, P. R., \& Montiel, P. J. (2008). Development Macroeconomics. New Jersey: Princeton University Press.

Akerlof, G. A., \& Yellen, J. L. (1986). Efficiency wage models of the labor market. New York: Cambridge University Press.

Aron, J., Macdonald, R., \& Muellbauer, J. (2014). Exchange rate pass-through in developing and emerging markets: A survey of conceptual, methodological and policy issues, and selected empirical findings. Journal of Development Studies, 50 (1), 101-143.

Bacchetta, P., \& Wincoop, E. (2003). Why do consumer prices react less than import prices to exchange rates? Journal of the european economic association, $1(2-3), 662-670$.

Ball, L. M., \& Sheridan, N. (2004). Does inflation targeting matter? En B. S. Bernanke \& M. Woodford (Eds.). The Inflation-Targeting Debate (pp. 249-282). Chicago, Illinois: University of Chicago Press. Recuperado de http://www. nber.org/chapters/c9561.pdf

Barro, R. J., \& Gordon, D. B. (1983). Rules, discretion and reputation in a model of monetary policy. Journal of Monetary Economics, 12 (1), 101-121.

Burstein, A., Eichenbaum, M., \& Rebelo, S. (2005). Large Devaluations and the 
Real Exchange Rate. The Journal of Political Economy, 113 (4), 742-784.

Bussière, M., Saxena, S. C., \& Tovar, C. E. (2012). Chronicle of currency collapses: Reexamining the effects on output. Journal of International Money and Finance, 31 (4), 680-708.

Ca’Zorzi, M., Hahn, E., \& Sánchez, M. (2007). Exchange rate pass-through in emerging markets. The IUP Journal of Monetary Economics, (4), 84-102.

Cagan, P. (1956). The Monetary Dynamics of Hyper-inflations. En M. Friedman (Ed.). Studies in the Quantity Theory of Money (pp. 25-117). Chicago: Chicago University Press.

Céspedes, L. F., Chang, R., \& Velasco, A. (2014). Is inflation targeting still on target? The recent experience of Latin America. International Finance, 17 (2), 185-208.

Dornbusch, R. (1980). Open economy macroeconomics. New York: Basic Books.

Dornbusch, R., \& Fischer, S. (1993). Moderate inflation. The World Bank Economic Review, 7 (1), 1-44.

Feenstra, R. C., \& Kendall, J. D. (1997). Pass-through of exchange rates and purchasing power parity. Journal of International Economics, 43 (12), 237-261.

Fischer, S., Sahay, R., \& Végh, C. A. (2002). Modern Hyper- and High Inflations. Journal of Economic literature, 40 (3), 837-880.

Frankel, J., Parsley, D., \& Wei, S.-J. (2012). Slow pass-through around the world: a new import for developing countries? Open Economies Review, 23 (2), 213-251.

Frenkel, R. (1986). Salarios e inflación en América Latina. Resultados de investigaciones recientes en la Argentina, Brasil, Colombia, Costa Rica y Chile. Desarrollo Económico, 25 (100), 587-622.

Frenkel, R. (1989). El régimen de alta inflación y el nivel de actividad. CEDES, Working paper $\mathrm{N}^{\circ}{ }^{2}$ 26. Recuperado de http://www.itf.org.ar/ingles/pdf/ documentos/51-1989.pdf

Frenkel, R., \& Rapetti, M. (2010). A concise history of exchange rate regimes in Latin America. Washington, D. C.: Center for Economic Policy and Research.

Froot, K., \& Rogoff, K. (Eds.) (1995). Chapter 32: Perspectives on PPP and longrun real exchange rates. En Handbook of International Economics, vol. 3. (1647-1688).

Goldberg, P. K., \& Hellerstein, R. (2008). A structural approach to explaining incomplete exchange-rate pass-through and pricing-to-market. The American Economic Review, 98 (2), 423-429.

Gonçalves, C. E. S., \& Salles, J. M. (2008). Inflation targeting in emerging economies: What do the data say? Journal of Development Economics, 85 (1), 312-318.

Hammond, G. (2012). State of the art of inflation targeting. In Handbook No. 29. London: Centre for Central Banking Studies, Bank of England. Recuperado de http://www.bankofengland.co.uk/education/Documents/ccbs/hand- 
books/pdf/ccbshb29.pdf

Hausman, J. A., Abrevaya, J., \& Scott-Morton, F. M. (1998). Misclassification of the dependent variable in a discrete-response setting. Journal of Econometrics, 87 (2), 239-269.

Heymann, D. (1986). Tres ensayos sobre inflación y políticas de estabilización. CEPAL, Working Paper N. ${ }^{\circ}$ 18. Recuperado de http://repositorio.cepal.org/ handle/11362/28518

Heymann, D., \& Leijonhufvud, A. (1995). High inflation: The Arne Ryde memorial lectures. Oxford: Clarendon Press.

Hicks, J. (1989). A market theory of money. London: Oxford University Press.

Horrace, W. C., \& Oaxaca, R. L. (2006). Results on the bias and inconsistency of ordinary least squares for the linear probability model. Economics Letters, 90 (3), 321-327.

Ito, T., \& Sato, K. (2008). Exchange Rate Changes and Inflation in Post-Crisis Asian Economies: Vector Autoregression Analysis of the Exchange Rate PassThrough. Journal of Money, Credit and Banking, 40 (7), 1407-1438.

McDonald, I. M., \& Solow, R. M. (1981). Wage bargaining and employment. The American Economic Review, 71 (5), 896-908.

Olivera, J. H. (1967). Aspectos dinámicos de la inflación estructural. Desarrollo Económico, 7 (27), 261-266.

Olivera, J. H. (1991). Equilibrio social, equilibrio de mercado e inflación estructural. Desarrollo Económico, 30 (120), 487-493.

Phelps, E. S. (1969). The New Microeconomics in Inflation and Employment Theory. The American Economic Review, 59 (2), 147-160.

Shambaugh, J. C. (2004). The Effect of Fixed Exchange Rates on Monetary Policy. The Quarterly Journal of Economics, 119 (1), 301-352.

Taylor, J. B. (2000). Low inflation, pass-through, and the pricing power of firms. European economic review, 44 (7), 1389-1408.

Tommasi, M. (1994). The Consequences of Price Instability on Search Markets: Toward Understanding the Effects of Inflation. The American Economic Review, 84 (5), 1385-1396.

Vera, L. (2014). Inflación estructural redux. Ensayos económicos, 69, 37-99.

Wooldridge, J. M. (2010). Econometric analysis of cross section and panel data. Cambridge, Massachusetts: The MIT press.

(C) 2017 por los autores; licencia otorgada a la revista Estudios económicos. Este artículo es de acceso abierto y distribuido bajo los términos y condiciones de una licencia Atribución-No Comercial 3.0 Unported (CC BY-NC 3.0) de Creative Commons. Para ver una copia de esta licencia, visite http://creativecommons.org/ licenses/by-nc/3.0/ 